\title{
The effect of obesity and type 2 diabetes (T2DM) on human metabolite rhythms
}

\author{
Cheryl Isherwood, Jonathan D. Johnston and Debra J. Skene \\ Chronobiology, Faculty of Health and Medical Sciences, University of Surrey, Guildford, GU2 $7 X H$
}

Obesity and type 2 diabetes (T2DM) have been associated with increased circulating concentrations of acylcarnitines ${ }^{(1)}$ and branched chain amino acids (BCAA) (isoleucine, leucine, and valine) in single time point studies ${ }^{(2)}$. Investigating 24 h daily rhythms (peak time and amplitude) of these metabolites in obesity and T2DM would help direct future study into any circadian clock-related mechanisms behind these changes.

The hypothesis that weight and/or T2DM affect the concentration and rhythmicity of acylcarnitines and BCAA across a $24 \mathrm{~h}$ day was tested.

In a controlled laboratory study, hourly milkshakes (06:30-21:30 h) were given to 8 lean $(\mathrm{L}), 10$ overweight $(\mathrm{OW})$ and $7 \mathrm{OW}$ with T2DM men, aged 45-65 years, lying semi-recumbent and awake between 06:30-22:30 h (light period) and asleep between 22:30 $06: 30 \mathrm{~h}$ (dark period). Plasma samples $(2 \mathrm{~h}$ across $24 \mathrm{~h}$ ) were analysed using quantitative targeted LC/MS metabolomics (Biocrates, Austria).

Two-way ANOVA identified significant group differences with increased $24 \mathrm{~h}$ concentrations in propionylcarnitine (C3) (L vs OW and L vs T2DM, p <0.001), butyrylcarnitine (C4) (L vs OW and L vs T2DM, p < 0.001) and valerylcarnitine (C5) (L vs OW, $\mathrm{p}<0.001$ ) in OW and T2DM compared to the L group. C3 and C4 displayed significant $24 \mathrm{~h}$ rhythms in all groups (Fig. 1). C5 lost its rhythm in OW and T2DM. Weight and T2DM increased the $24 \mathrm{~h}$ concentrations of isoleucine $(\mathrm{L}$ vs $\mathrm{OW}$, p $=0.009$; $\mathrm{L}$ vs T2DM, p <0.001), leucine ( $\mathrm{L}$ vs OW, $\mathrm{p}=0.035 ; \mathrm{L}$ vs T2DM, p =0.010) and valine (L vs T2DM, p < 0.001) in the OW and T2DM groups compared to the L group. Isoleucine and valine displayed significant daily rhythms in the $\mathrm{L}$ and $\mathrm{OW}$ groups only (Fig. 1), with no significant daily rhythm for leucine in any group.
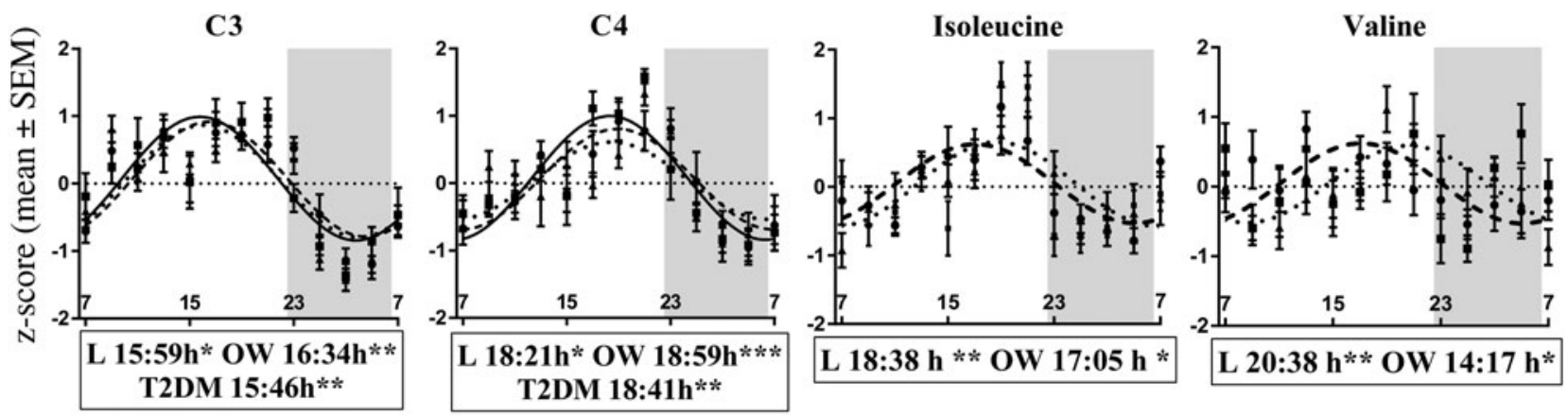

Time of day (hours)

Figure 1. $24 \mathrm{~h}$ rhythms of propionylcarnitine (C3), butyrylcarnitine (C4), valine and isoleucine. Where a significant daily cosine rhythm was expressed $(* \mathrm{p}<0.05, * \mathrm{p}<0.01, * * * \mathrm{p}<0.005)$, the peak time is shown below each graph. The L group is represented by a dotted line; OW a dashed line, and T2DM a solid line.

The increased concentrations of the short-chain acylcarnitines, C3, C4 and C5, and BCAA in obesity and T2DM support previous studies. $\mathrm{C} 3$ and $\mathrm{C} 4$ retained their $24 \mathrm{~h}$ rhythm in all groups although $\mathrm{C} 5$ rhythmicity was lost in OW and T2DM. Isoleucine and valine lost rhythmicity in T2DM. Our findings likely reflect disruption of normal energy metabolism in OW and/or T2DM. The rhythms observed may result from the complex interaction between metabolism in the fed and fasting state and/or circadian timing.

This work was funded by Diabetes-UK (grant 08/0003607), EU FP7-HEALTH-2011 EuRhythDia (grant 278397), BBSRC (grant BB/I019405/1 and grant BB/D526853/1) and Stockgrand Ltd.

1. Schooneman MG, Vaz FM, Houten SM, et al., (2013) Diabetes 62, 1-8

2. Newgard CB, An J, Bain JR, et al. (2009) Cell Metab 9, 311-326 